\title{
PREFACE
}

\section{Chloroplast transport and import}

\author{
Steven M. Theg ${ }^{1}$
}

Received: 3 October 2018 / Accepted: 3 October 2018 / Published online: 5 November 2018

(c) Springer Nature B.V. 2018

As is well known to readers of this Special Issue, chloroplasts possess their own genetic material and are capable of a limited amount of autonomous protein synthesis. Nevertheless, more than $95 \%$ of the protein complement of chloroplasts comes from nucleus-encoded genes. These genes are transcribed in the nucleus, translated on $80 \mathrm{~S}$ ribosomes in the plant cell cytoplasm, and taken up post-translationally into the organelle, where they are sorted to one or more of six compartments. The manner in which this is accomplished has been the subject of intense research efforts since it was first described in the classic paper by Chua and Schmidt (1978). As expected in any scientific endeavor, our understanding of these and related events did not arise quickly and linearly, but grew piece by piece with many fits and starts. And of course, it is by no means complete today. That said, there is much that we do know about these processes, and this Special Issue was conceived as a means to collect much of this information under one title.

In addition to possessing a complex ultrastructure from a targeting perspective, chloroplasts possess at least four, and probably more, separate protein transport machineries. Our initial information about the protein machinery responsible for protein import into the stroma derives from isolation of proteins in contact with importing substrates (Akita et al. 1997; Kessler et al. 1994; Hirsch et al. 1994). From these initial findings, an extensive knowledge of the so-called Toc and Tic translocons has emerged. The article by Bölter presents an overview of the state of knowledge surrounding these translocons and their mechanisms of action, touching on unknown questions still requiring resolution.

Protein targeting from the stroma into and across the thylakoid membrane is achieved via three energy-dependent transport machineries. The cpSRP pathway, originally identified in the Hoffman and Cline laboratories (Li et al.

Steven M. Theg

smtheg@ucdavis.edu

1 Department of Plant Biology, University of California, Davis, Davis, CA 95616, USA
1995), has been the subject of extensive investigation in the intervening years. The latest thinking about this pathway is described in the article by Ziehe, Dünschede, and Schünemann. Two additional transport machineries, both arguably first described in the Cline laboratory (Cline et al. 1993; Yuan et al. 1994), take proteins into and across the thylakoid membrane. The cpSec pathway is the subject of the article by Fernandez, and cpTat pathway is fully described by New, Ma, and Dabney-Smith. Our understanding of the targeting of proteins to the chloroplast outer envelope membrane is less complete than for these other systems, and this topic is reviewed in the article by Day and Theg. The latest transport machinery to be described (Skalitzky et al. 2011), the Sec2 system involved in protein traffic to the inner envelope membrane, is discussed in the article by Fernandez.

A number of relatively newer topics are described in this Special Issue that are often overlooked when dealing with targeting in chloroplasts. These include the newly discovered regulation of chloroplast protein import during plant and leaf development (Chu and $\mathrm{Li}$ ), and the dual targeting of proteins to chloroplasts and mitochondria (Sharma, Bennewitz, and Klösgen), a phenomenon we originally thought was restricted to a few proteins but which we are now learning is more widespread.

Finally, two related topics not usually part of the discussion of chloroplast targeting events are included here. The article by LeBrant, Barness, and Roston presents an overview of the acquisition of lipids in chloroplasts, from fatty acid synthesis in the stroma to their export to the ER and subsequent re-entry into and distribution within chloroplasts. Finally, the poorly characterized phenomenon of vesicle traffic to and within chloroplasts is discussed in the article by Lindquist and Aronsson, with a comprehensive description of the proteins implicated in such vesicle traffic.

Topics were chosen for this Special Issue to give a broad perspective of the field, and articles were written by researchers who each were major contributors to their chosen topics. The result is an issue that contains a wealth of information about the transport of proteins and lipids into and within plastids, useful to both generalists and teachers 
wanting to get an overview of the field, and to specialists looking for in depth treatment of the different topics.

I wish to thank all of the authors for their excellent contributions, and the reviewers for their many thoughtful suggestions for improvements. I also thank Terry Bricker (Editorin-Chief) for the invitation to edit this Special Issue, and for our many helpful discussions concerning its contents. Support from the Division of Chemical Sciences, Geosciences, and Biosciences, Office of Basic Energy Sciences of the U.S. Department of Energy through Grant DE-FG0203ER 15405 during the preparation of this Special Issue is gratefully acknowledged.

\section{References}

Akita M, Nielsen E, Keegstra K (1997) Identification of protein transport complexes in the chloroplastic envelope membranes via chemical cross-linking. J Cell Biol 136(5):983-994
Chua NH, Schmidt GW (1978) Post-translational transport into intact chloroplasts of a precursor to the small subunit of ribulose-1,5-bisphosphate carboxylase. Proc Natl Acad Sci USA 75(12):6110-6114

Cline K, Henry R, Li C, Yuan J (1993) Multiple pathways for protein transport into or across the thylakoid membrane. EMBO J 12(11):4105-4114

Hirsch S, Muckel E, Heemeyer F, Von Heijne G, Soll J (1994) A receptor component of the chloroplast protein translocation machinery. Science 266:1989-1992

Kessler F, Blobel G, Patel HA, Schnell DJ (1994) Identification of two GTP-binding proteins in the chloroplast protein import machinery. Science 266:1035-1039

Li X, Henry R, Yuan J, Cline K, Hoffman NE (1995) A chloroplast homologue of the signal recognition particle subunit SRP54 is involved in the posttranslational integration of a protein into thylakoid membranes. Proc Natl Acad Sci USA 92(9):3789-3793

Skalitzky CA, Martin JR, Harwood JH, Beirne JJ, Adamczyk BJ, Heck GR, Cline K, Fernandez DE (2011) Plastids contain a second sec translocase system with essential functions. Plant Physiol 155(1):354-369. https://doi.org/10.1104/pp.110.166546

Yuan J, Henry R, McCaffery M, Cline K (1994) SecA homolog in protein transport within chloroplasts: evidence for endosymbiont derived sorting. Science 266:796-798 